\title{
Might hypovitaminosis D aggravate endothelial dysfunction-related increases in arterial stiffness seen in patients with hypertension and type 2 diabetes? Reply to Boucher BJ [letter]
}

\author{
R. M. Bruno • L. Ghiadoni • G. Penno • S. Taddei • \\ S. Del Prato
}

Received: 10 July 2012 / Accepted: 18 July 2012 / Published online: 4 September 2012

(C) Springer-Verlag 2012

\begin{abstract}
Abbreviations
25(OH)D 25-Hydroxyvitamin D

FMD Flow-mediated dilation
\end{abstract}

To the Editor: The role of vitamin $\mathrm{D}$ in vascular disease and function is a hot, emerging issue in cardiovascular medicine [1]. Prospective cohort studies have identified 25hydroxyvitamin D (25-[OH]D) level as an independent predictor for cardiovascular disease whereas trials on vitamin D supplementation have yielded conflicting results, possibly because of great heterogeneity in the study populations and variability in the supplementation dose [1]. Nonetheless, a number of pathophysiological studies support the hypothesis that vitamin D may be involved in vascular homeostasis and in the modulation of endothelial function through multiple mechanisms, including effects on the renin-angiotensin system, vascular inflammation and oxidative stress [2-4]. In a study enrolling 554 healthy individuals, vitamin D insufficiency was associated with increased arterial stiffness and endothelial dysfunction (using brachial artery flow-mediated dilation [FMD]) in the conductance and resistance blood vessels, irrespective of

R. M. Bruno $(\bowtie) \cdot$ L. Ghiadoni $\cdot$ S. Taddei

Department of Internal Medicine, University of Pisa,

Via Roma 67,

56126 Pisa, Italy

e-mail: rosam.bruno@gmail.com

R. M. Bruno

CNR - Institute of Clinical Physiology,

Pisa, Italy

G. Penno $\cdot$ S. Del Prato

Department of Endocrinology and Metabolism, University of Pisa,

Pisa, Italy traditional risk burden [5]. Furthermore, in 280 type 2 diabetic individuals, serum $25(\mathrm{OH}) \mathrm{D}$ levels were associated with FMD and circulating $\mathrm{CD} 133^{+} / \mathrm{KDR}^{+}$endothelial progenitor cells. The latter suggests that vitamin $\mathrm{D}$ deficiency may contribute to endothelial dysfunction in patients with type 2 diabetes [6]. A few interventional studies have suggested that vitamin D supplementation could ameliorate endothelial function in diabetic patients [2] as well as in vitamin D deficient young healthy volunteers [7]. A final conclusion is hard to draw from these studies because of small sample size and concomitant confounders. Vitamin D deficiency has been associated with increased risk for development of diabetes in humans. Moreover, experimental studies confirm a role for vitamin D in glucose homeostasis, although vitamin D supplementation is not associated with improved glucose control in diabetic patients [8]. All these observations seem to fit well with the hypothesis brought up by Boucher [9]. She suggests, indeed, that hypovitaminosis D could be responsible for the incremental detriment in endothelial function that we have documented [10] in hypertensive patients with diabetes compared with those with normal glucose tolerance. Although intriguing, this explanation remains highly speculative and cannot be demonstrated in our study population as, unfortunately, we did not provide for vitamin D serum levels to be measured at the time of study planning. Nonetheless, we must admit that this is a factor we will take into full account in future investigation as evidence for a role of vitamin D in vasculature function is mounting. In summary, we appreciate Dr Boucher's suggestion and tend to agree with her on the importance of considering vitamin $\mathrm{D}$ as a potential factor involved in vasculature function and protection. Her suggestion, along with our findings, prompt for more studies generating high-quality data. These studies will require adequate sample size, allowing adjustment for multiple confounders, in order to establish whether vitamin D deficiency 
can be considered a causative factor in vascular disease or a mere marker of poor health status.

Contribution statement All authors were responsible for the conception and design of the manuscript, drafting the article and revising it critically for important intellectual content. All authors approved the final version of the letter.

Duality of interest The authors declare that there is no duality of interest associated with this manuscript.

\section{References}

1. McGreevy C, Williams D (2011) New insights about vitamin D and cardiovascular disease: a narrative review. Ann Intern Med 155:820-826

2. Sugden JA, Davies JI, Witham MD, Morris AD, Struthers AD (2008) Vitamin D improves endothelial function in patients with type 2 diabetes mellitus and low vitamin D levels. Diabet Med 25:320-325

3. Jablonski KL, Chonchol M, Pierce GL, Walker AE, Seals DR (2011) 25-Hydroxyvitamin D deficiency is associated with inflammation- linked vascular endothelial dysfunction in middle-aged and older adults. Hypertension 57:63-69

4. Dong J, Wong SL, Lau CW et al (2012) Calcitriol protects renovascular function in hypertension by down-regulating angiotensin II type 1 receptors and reducing oxidative stress. Eur Heart J. doi:10.1093/eurheartj/ehr459

5. Al Mheid I, Patel R, Murrow J et al (2011) Vitamin D status is associated with arterial stiffness and vascular dysfunction in healthy humans. J Am Coll Cardiol 58:186-192

6. Yiu YF, Chan YH, Yiu KH et al (2011) Vitamin D deficiency is associated with depletion of circulating endothelial progenitor cells and endothelial dysfunction in patients with type 2 diabetes. J Clin Endocrinol Metab 96:E830-E835

7. Tarcin O, Yavuz DG, Ozben B et al (2009) Effect of vitamin D deficiency and replacement on endothelial function in asymptomatic subjects. J Clin Endocrinol Metab 94:4023-4030

8. Pittas AG, Chung M, Trikalinos T et al (2010) Systematic review: vitamin D and cardiometabolic outcomes. Ann Intern Med 152: 307-314

9. Boucher BJ (2012) Might hypovitaminosis D aggravate endothelial dysfunction-related increases in arterial stiffness seen in patients with hypertension and type 2 diabetes? Diabetologia doi:10.1007/s00125-012-2705-z

10. Bruno RM, Penno G, Daniele G et al (2012) Type 2 diabetes mellitus worsens arterial stiffness in hypertensive patients through endothelial dysfunction. Diabetologia 55:1847-1855 